\title{
Fatal Chromobacterium violaceum septicaemia in northern Laos, a modified oxidase test and post-mortem forensic family G6PD analysis
}

\author{
Günther Slesak ${ }^{1}$, Phouvieng Douangdala ${ }^{2}$, Saythong Inthalad ${ }^{1}$, Joy Silisouk ${ }^{3}$, \\ Manivanh Vongsouvath ${ }^{3}$, Amphonesavanh Sengduangphachanh ${ }^{3}$, \\ Catrin E Moore ${ }^{3,4}$, Mayfong Mayxay ${ }^{3,5}$, Hiroyuki Matsuoka ${ }^{6}$ and \\ Paul N Newton*3,4
}

\begin{abstract}
Address: ${ }^{1}$ SFE Medical Project, P.O. Box 56, Luang Namtha, Lao People's Democratic Republic, ${ }^{2}$ Luang Namtha Provincial Hospital, Luang Namtha, Lao People's Democratic Republic, ${ }^{3}$ Wellcome Trust-Mahosot Hospital-Oxford Tropical Medicine Research Collaboration, Microbiology Laboratory, Mahosot Hospital, Vientiane, Lao People's Democratic Republic, ${ }^{4}$ Centre for Clinical Vaccinology and Tropical Medicine, Churchill Hospital, University of Oxford, Oxford, England, UK, ${ }^{5}$ Faculty of Post-Graduate Studies and Research, University of Health Sciences, Vientiane, Lao PDR and ${ }^{6}$ Division of Medical Zoology, Department of Infection and Immunity, Jichi Medical University, Japan

Email: Günther Slesak - guentherslesak@web.de; Phouvieng Douangdala - phouviengddl@yahoo.com;

Saythong Inthalad - saythongi@yahoo.com; Joy Silisouk - jsirisouk@yahoo.com; Manivanh Vongsouvath - manivanh100@ hotmail.com; Amphonesavanh Sengduangphachanh - ampkg@live.com; Catrin E Moore - catrin@tropmedres.ac;

Mayfong Mayxay - mayfong@tropmedres.ac; Hiroyuki Matsuoka - hiroyuki@jichi.ac.jp; Paul N Newton* - paul@tropmedres.ac

* Corresponding author
\end{abstract}

Published: 29 July 2009

Annals of Clinical Microbiology and Antimicrobials 2009, 8:24 doi:10.1 I86/1476-07| I-8-24

This article is available from: http://www.ann-clinmicrob.com/content/8/I/24

(C) 2009 Slesak et al; licensee BioMed Central Ltd.

This is an Open Access article distributed under the terms of the Creative Commons Attribution License (http://creativecommons.org/licenses/by/2.0), which permits unrestricted use, distribution, and reproduction in any medium, provided the original work is properly cited.
Received: 5 April 2009

Accepted: 29 July 2009

\begin{abstract}
Background: Chromobacterium violaceum is a Gram negative facultative anaerobic bacillus, found in soil and stagnant water, that usually has a violet pigmented appearance on agar culture. It is rarely described as a human pathogen, mostly from tropical and subtropical areas.

Case presentation: A 53 year-old farmer died with Chromobacterium violaceum septicemia in Laos. A modified oxidase method was used to demonstrate that this violacious organism was oxidase positive. Forensic analysis of the glucose-6-phosphate dehydrogenase genotypes of his family suggest that the deceased patient did not have this possible predisposing condition.

Conclusion: $C$. violaceum infection should be included in the differential diagnosis in patients presenting with community-acquired septicaemia in tropical and subtropical areas. The apparently neglected but simple modified oxidase test may be useful in the oxidase assessment of other violetpigmented organisms or of those growing on violet coloured agar.
\end{abstract}

\section{Background}

Chromobacterium violaceum is a Gram negative facultative anaerobic bacillus, found in soil and stagnant water, that usually has a violet pigmented appearance on agar culture. Since its discovery as a human pathogen in 1927 in
Malaysia [1] only 150 human cases have been reported worldwide, mostly from tropical and subtropical areas. $C$. violaceum septicaemia has been described in Thailand, with two patients from a province adjacent to Laos [2] and Vietnam $[3,4]$ but, to our knowledge, this is the first 
patient described in Laos. The disease typically starts with a localized skin infection or localized lymphadenitis after contact with stagnant water or soil and progresses to fulminating septicaemia with necrotizing metastatic lesions and multiple abscesses in the liver, lung, spleen, skin, lymph nodes, and brain, resulting in fatal multiorgan failure [1-10]. The mortality from disseminated C. violaceum infection has been reported to be $60-80 \%$ [1-10]. However, the optimal treatment has not been established combination therapy with co-trimoxazole, chloramphenicol, carbapenems, or fluoroquinolones has been suggested $[2,7,9]$. Resistance to penicillins and cephalosporins has been commonly reported $[3,9,11]$ and makes therapy while microbiology results are awaited difficult as both antibiotics are commonly used for empirical therapy of septicemia.

\section{Case presentation}

A 53-year-old Tai Dam [12] Lao farmer was admitted in August 2008 at the Provincial Hospital of Luang Namtha, northern Lao PDR (Laos), with 2 days of fever, chills, severe headaches, nausea and vomiting, and a cough productive of white sputum. He had been healthy apart from serious leg injuries from a relict bomb explosion 6 years previously. On admission he was alert and orientated, normotensive, without neck stiffness, abdominal tenderness, lymphadenopathy, hepatosplenomegaly, pallor or jaundice but was febrile $\left(39.7^{\circ} \mathrm{C}\right)$ with crepitations audible at the left lung base. He had no rash but old scars on his thighs from the bomb injury and minor scars on his lower legs and flanks from insect bites. He was not known to contain shrapnel. With suspected community-acquired pneumonia he was started on oral amoxicillin (1 g twice daily) and paracetamol. With an increase of his fever to $40.2^{\circ} \mathrm{C}$, metamizole ( $1 \mathrm{~g}$ twice daily IV) was added. On day 2 his full blood count showed normochromic anaemia (Hct 18\%, Hb 6 g/dL, MCHC 33 g/dL, leucocytes 7.5 $10 \%$ L, neutrophils 70\%, lymphocytes 30\%). Malaria smear and HRP-2 rapid test for Plasmodium falciparum malaria (Paracheck ${ }^{\mathrm{TM}}$, Orchid Industries, Goa, India) were negative. An abdominal ultrasound demonstrated a diffuse enlarged liver, without focal lesions, and mild splenomegaly. A chest radiograph showed a slightly enlarged heart but no infiltrations. The patient developed upper abdominal and lower back pain and increasing dyspnoea and was pale and jaundiced with a respiratory rate of $42 / \mathrm{min}$, temperature $39^{\circ} \mathrm{C}$, blood pressure $90 / 60$ $\mathrm{mmHg}$, heart rate irregular $120 / \mathrm{min}$, bibasal inspiratory crepitations, tender hepatomegaly $(1 \mathrm{~cm}$ below costal margin in mid-clavicular line) with axillary and inguinal lymphadenopathy. As community-acquired septicaemia or leptospirosis were suspected, therapy was changed to ceftriaxone ( $1 \mathrm{~g}$ twice daily) and he was given a blood transfusion. However, he deteriorated with worsening abdominal pain and distension, dyspnoea, central cyano- sis with cold extremities, coma (GCS 7/15) and a disseminated maculopapular rash with petechiae on arms and trunk and prolonged bleeding at intravenous injection sites. On day 3 he became apnoeic and could not be resuscitated.

Blood cultures from admission and day 2, sent by plane to Vientiane [13,14], grew Chromobacterium violaceum (Gram negative rods, catalase positive, Voges-Proskauer test negative, violacious colour on agar, API 20 NE (bioMerieux, France) 99.3\% agreement). As the C. violaceum were pigmented violet conventional oxidase tests could not be interpreted and the ingenious but apparently neglected method of Dhar \& Johnson [15] was used to demonstrate that the organism was oxidase positive (Fig. 1). The organism was sensitive to gentamicin, chloramphenicol, ofloxacin, ciprofloxacin, co-trimoxazole, imipenem, and resistant to ampicillin, ceftriaxone, and ceftazidime by disc diffusion testing (using NCCLS criteria for Vibrio or Enterobacteracea). Etest (AB Biodisk, Solna, Sweden) demonstrated MICs of $0.023,4$ and $>32 \mathrm{ug} / \mathrm{L}$ against ofloxacin, chloramphenicol and ceftriaxone, respectively. After his death his family was re-interviewed regarding possible risk factors. He had been pale with mild jaundice since childhood and when aged $\sim 12$ years was said to have had a big spleen. One week before the onset of fever he had gone to rice fields and fishing and slept at a market where he noted an axillary leech. The bite wound did not become overtly infected.

Glucose-6-phosphate dehydrogenase (G6PD) deficiency has been suggested as a risk factor for $C$. violaceum infection $[7,16]$ but the aetiological diagnosis was only made after the patient's death and no blood samples were available for G6PD testing. Therefore, with their informed consent, his wife and 4 daughters were tested for G6PD deficiency, using finger prick blood samples on filter paper, by quantitative spectrophotometric analysis (WST8 method [17]) and sequencing of the G6PD gene in the $\mathrm{X}$-chromosome [18]. The patient's wife and 3 of the 4 tested daughters had a substitution of G to A at the 1388 position in G6PD gene, signifying carriage of G6PD Kaiping. Since one of the daughters had wild type X-chromosomes with normal G6PD activity the patient evidently did not have G6PD deficiency.

Our patient did not have a primary skin infection. However, he had a leech bite a week before onset of fever which may or not have been significant - as far as we are aware this organism has not been described from the leech gastrointestinal tract. The patient's striking anaemia, severe abdominal and lower back pain, tachycardia, and jaundice in combination with a history of pallor and splenomegaly in childhood could be explained by an acute intravascular haemolysis due to underlying G6PD 

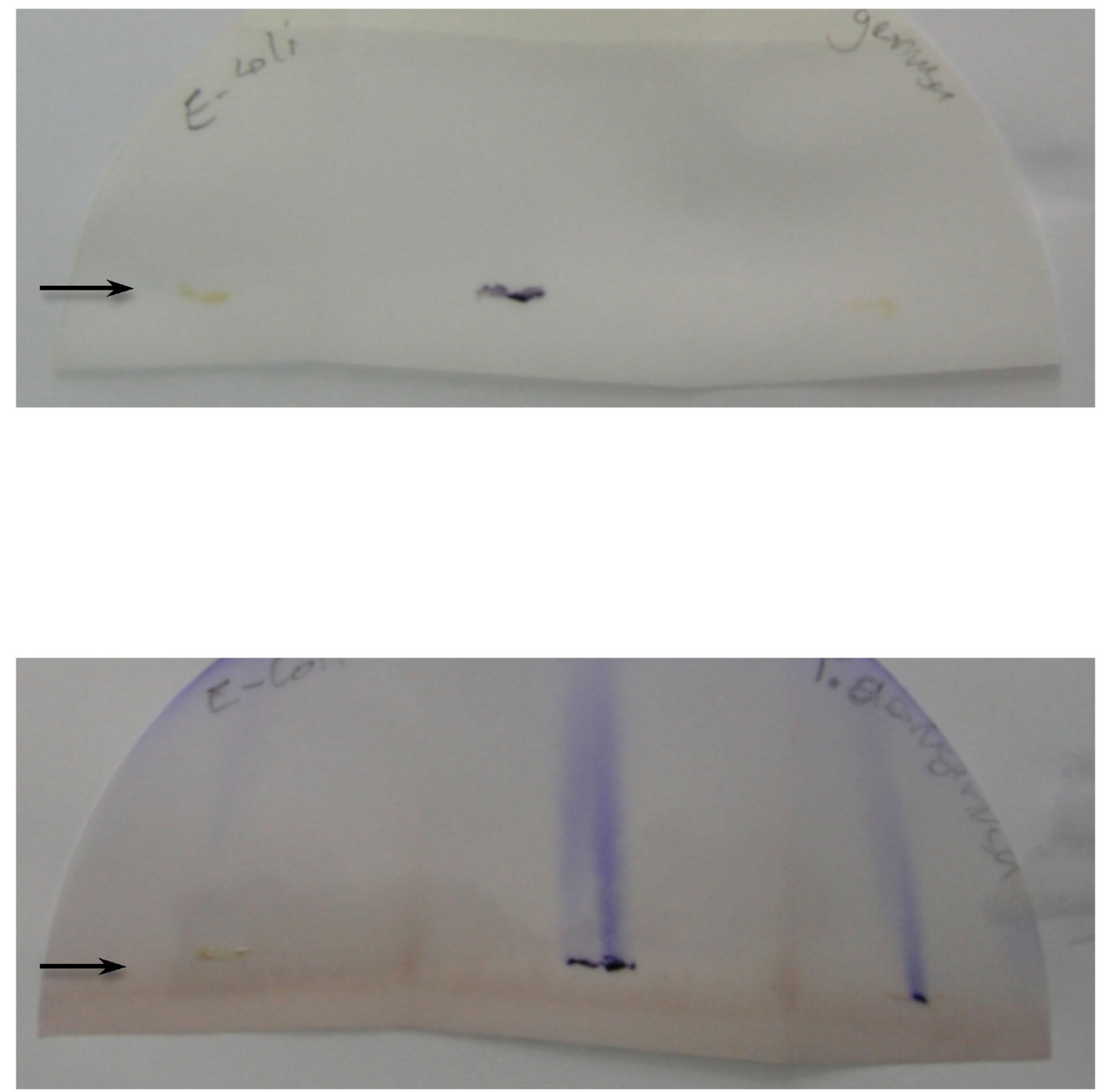

\section{Figure I}

Oxidase test adapted from Dhar \& Johnson [15]. Smears (arrows) of organisms were applied to two Whatman 3 M filterpapers folded, vertically, into three. The filter papers were placed vertically in Petri dishes so that the solution within soaked vertically across the smears of organisms. Top panel: filterpaper stood in $10 \mathrm{ml}$ distilled water in a Petri dish, bottom panel: filterpaper stood in $10 \mathrm{ml} \mathrm{I \%} \mathrm{aqueous} \mathrm{solution} \mathrm{of} \mathrm{N,} \mathrm{N,} \mathrm{N',} \mathrm{N'-tetramethyl-I,} \mathrm{4-phenylene-diamine} \mathrm{dihydrochloride} \mathrm{(Fluka}$ 87890, Czech Republic) in a Petri dish. The organisms smeared were left E. coli ATCC 25922 (negative control), centre C. violaceum isolate from patient and right $P$. aeruginosa ATCC 27853 (positive control). Water (top panel) failed to produce any streaks whilst N,N,N',N'-tetramethyl-I, 4-phenylene-diamine dihydrochloride (bottom panel) led to pronounced vertical flame-like streaks across smears of $C$. violaceum and $P$. aeruginosa within 5 minutes, indicating oxidase test positivity. The flamelike streaks had faded by the next day. The profound violet color of the $C$. violaceum smear is clearly visible. 
deficiency, possibly precipitated by metamizole (dipyrone), which is contraindicated in G6PD deficiency [19]. Laos has a high prevalence of G6PD deficiency (15-26\% of males hemizygous $[20,21])$. In view of G6PD being a possible risk factor for both his infection and haemolysis we performed forensic analysis of the surviving members of his family. However, this demonstrates, assuming correct blood relationships, that he could not have had G6PD deficiency. G6PD Kaiping is a common mutation in neighboring China [22].

Melioidosis (Burkholderia pseudomallei) is a common and frequently fatal condition in Northeast Thailand and the Mekong valley of Laos [14] but has, so far, not been described from the highlands of northern Laos and it may not occur in the soil there. C. violaceum and melioidosis resemble each other with reservoirs in soil and water, clinical presentation, resistance to antibiotics commonly used to empirically treat septicaemia, high mortality and the need for prolonged oral antibiotic eradication therapy. However, as C. violaceum is frequently resistant to cephalosporins, such as ceftazidime $[5,9]$, commonly used to treat melioidosis, awareness of $C$. violaceum as an alternative diagnosis is important [2]. The two organisms can be confused in the laboratory and both are oxidase positive [23]. The ingenious method of Dhar \& Johnson [15] was used to determine the oxidase status of the organism this apparently neglected but simple technique may be useful in the oxidase assessment of other violet-pigmented organisms or of those growing on violet colored agar. A similar method was described [24] after that of Dhar \& Johnson [15]. Conventional oxidase testing of very young or anaerobic $C$. violaceum cultures, which are usually non-pigmented, have also been used but the evidence available suggests that they may not be accurate $[6,24]$.

\section{Conclusion}

C. violaceum infection should be included in the differential diagnosis of patients presenting with communityacquired septicaemia in tropical and subtropical areas, especially with a history of contact with soil and stagnant water, and is an important alternative diagnosis for patients presenting with a melioidosis-like syndrome.

\section{Competing interests}

The authors declare that they have no competing interests.

\section{Authors' contributions}

GS, PD, SI were the attending physicians who looked after the patient. JS, MV, PNN, CEM and MM preformed the microbiological examinations. PD, GS did the home visits of the patient's family and HM the G6PD assays and sequencing. GS and PNN wrote the first draft and all authors revised it. All authors have read an approved the final version.

\section{Consent}

Written informed consent was obtained from the patient's family for publication of this case report and the forensic investigation.

\section{Financial support}

We thank the Wellcome Trust of Great Britain for financial support of the microbiology testing and SFE for the logistical support of the specimen transport.

\section{Acknowledgements}

We thank Rattanaphone Phetsouvanh, Phonlavanh Phouminh and colleagues at Mahosot Hospital for their assistance and Claude Gauthier and colleagues for their thorough review of the patient's chest radiography. We are very grateful for all the detailed information provided by the patient's family and to Sharon Peacock for helpful comments.

\section{References}

I. Sneath PH, Whelan JP, Bhagwan Singh R, Edwards D: Fatal infection by Chromobacterium violaceum. Lancet 1953, 265:276-277.

2. Jitmuang A: Human Chromobacterium violaceum infection in Southeast Asia: case reports and literature review. Southeast Asian J Trop Med Public Health 2008, 39:452-60.

3. Baker S, Campbell JI, Stabler R, Nguyen HVM, To DS, Nguyen DV, Farrar J: Fatal wound infection caused by Chromobacterium violaceum in Ho Chi Minh City, Vietnam. J Clin Microbiol 2008, 46:3853-3855.

4. Ognibene AJ, Thomas E: Fatal infection due to Chromobacterium violaceum in Vietnam. Am J Clin Pathol I970, 54:607-I0.

5. Chang CY, Lee YT, Liu KS, Wang YL, Tsao SM: Chromobacterium violaceum infection in Taiwan: a case report and literature review. Microbiol Immunol Infect 2007, 40:272-275.

6. Lee J, Kim JS, Nahm CH, Choi JW, Kim J, Pai SH, Moon KH, Lee K, Chong $Y$ : Two cases of Chromobacterium violaceum infection after injury in a subtropical region. J Clin Microbiol 1999, 37:2068-70.

7. Sirinavin S, Techasaensiri C, Benjaponpitak S, Pornkul R, Vorachit M: Invasive Chromobacterium violaceum infection in children: case report and review. Pediatr Infect Dis J 2005, 24:559-56 I.

8. Teoh AYB, Hui M, Ngo KY, Wong J, Lee KF, Lai PBS: Fatal septicaemia from Chromobacterium violaceum: case reports and review of the literature. Hong Kong Med J 2006, I 2:228-23I.

9. Ti TY, Tan WC, Chong APY, Lee EH: Nonfatal and fatal infections caused by Chromobacterium violaceum. Clin Inf Dis 1993, I 7:505-507.

10. Victorica B, Baer H, Ayoub EM: Successful treatment of systemic Chromobacterium violaceum infection. JAMA 1974, 230:578-580.

II. Aldridge KE, Valainis GT, Sanders CV: Comparison of the in vitro activity of ciprofloxacin and 24 other antimicrobial agents against clinical strains of Chromobacterium violaceum. Diagn Microbiol Infect Dis 1988, 10:31-39.

12. Chazée L: The Peoples of Laos. Rural and Ethnic Diversities Bangkok, White Lotus Co. Ltd; 2002.

13. Blacksell SD, Khounsy S, Phetsouvanh R, Newton PN: A simple and inexpensive container for the transport of biological specimens in limited resource situations. Trans $R$ Soc Trop Med Hyg 2006, 100:84-86.

14. Phetsouvanh R, Phongmany S, Soukaloun D, Rasachak B, Soukhaseum V, Soukhaseum S, Frichithavong K, Khounolatha S, Pengdee B, Phiasakha K, Chu K, Luansay K, Rattanavong S, Sisouk K, Keolouangkot V, Mayxay M, Ramsay A, Blacksell SD, Campbell J, Martinez-Aussel B, Heuanvongsy MM, Bounxouai B, Thammavong C, Syhavong B, Strobel M, Peacock SJ, White NJ, Newton PN: Causes Of CommunityAcquired Bacteremia And Patterns Of Antimicrobial Resistance In Vientiane, Laos. Am J Trop Med Hyg 2006, 75:978-985. 
15. Dhar SK, Johnson R: The oxidase activity of chromobacterium. J Clin Pathol 1973, 26:304-306.

16. Mamlok RJ, Mamlok V, Mills GC, Daeschner CW, Schmalstieg FC, Anderson DC: Glucose-6-phosphate dehydrogenase deficiency, neutrophil dysfunction and Chromobacterium violaceum sepsis. J Pediatr 1987, I I I:852-854.

17. Tantular IS, Kawamoto F: An improved, simple screening method for detection of glucose-6-phosphate dehydrogenase deficiency. Trop Med Int Health 2003, 8:569-574.

18. Matsuoka H, Thuan DT, van Thien H, Kanbe T, Jalloh A, Hirai M, Arai M, Dung NT, Kawamoto F: Seven different glucose-6-phosphate dehydrogenase variants including a new variant distributed in Lam Dong Province, southern Vietnam. Acta Medica Okayama 2007, 61:213-219.

19. Walz B, Riecken B: A young man with acute generalised jaundice and intermittent epigastric pain. Dtsch Med Wochenschr 2008, I33:129-132.

20. Cappellini MD, Fiorelli G: Glucose-6-phosphate dehydrogenase deficiency. Lancet 2008, 37 I:64-74.

21. Hsia YE, Miyakawa F, Baltazar J, Ching NS, Yuen J, Westwood B, Beutler $E$ : Frequency of glucose-6-phosphate dehydrogenase (G6PD) mutations in Chinese, Filipinos, and Laotians from Hawaii. Hum Genet 1993, 92:470-476.

22. Jiang $W, Y u$, Liu P, Geng Q, Chen L, Lin Q, Ren X, Ye W, He Y, Guo Y, Duan S, Wen J, Li H, Qi Y, Jiang C, Zheng Y, Liu C, Si E, Zhang $\mathrm{Q}$, Tian Q, Du C: Structure and function of glucose-6-phosphate dehydrogenase-deficient variants in Chinese population. Hum Genet 2006, I 1 9:463-478.

23. Inglis TJ, Chiang D, Lee GS, Chor-Kiang L: Potential misidentification of Burkholderia pseudomallei by API 20NE. Pathology 1998, 30:62-64.

24. Sivendra R, Lo HS, Lim KT: Identification of Chromobacterium violaceum: pigmented and non-pigmented strains. J Gen Microbiol 1975, 90:2|-3।.

Publish with Bio Med Central and every scientist can read your work free of charge

"BioMed Central will be the most significant development for disseminating the results of biomedical research in our lifetime. "

Sir Paul Nurse, Cancer Research UK

Your research papers will be:

- available free of charge to the entire biomedical community

- peer reviewed and published immediately upon acceptance

- cited in PubMed and archived on PubMed Central

- yours - you keep the copyright
BioMedcentral 